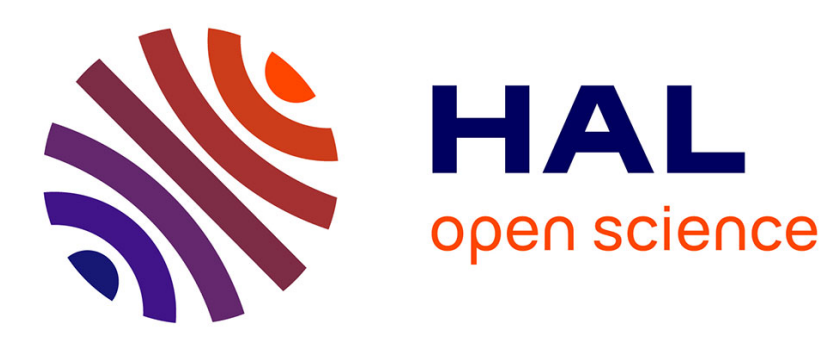

\title{
Variables d'arêtes et variables nodales dans la modélisation des champs magnétiques
}

\author{
B. Bandelier, F. Rioux-Damidau
}

\section{To cite this version:}

B. Bandelier, F. Rioux-Damidau. Variables d'arêtes et variables nodales dans la modélisation des champs magnétiques. Revue de Physique Appliquée, 1990, 25 (7), pp.605-612. 10.1051/rphysap:01990002507060500 . jpa-00246225

\section{HAL Id: jpa-00246225 https://hal.science/jpa-00246225}

Submitted on 1 Jan 1990

HAL is a multi-disciplinary open access archive for the deposit and dissemination of scientific research documents, whether they are published or not. The documents may come from teaching and research institutions in France or abroad, or from public or private research centers.
L'archive ouverte pluridisciplinaire HAL, est destinée au dépôt et à la diffusion de documents scientifiques de niveau recherche, publiés ou non, émanant des établissements d'enseignement et de recherche français ou étrangers, des laboratoires publics ou privés. 
Classification

Physics Abstracts

$41.10 \mathrm{~F}-89.20-02.70$

\title{
Variables d'arêtes et variables nodales dans la modélisation des champs magnétiques
}

\author{
B. Bandelier et F. Rioux-Damidau \\ Laboratoire de Génie Electrique de Paris, Ecole Supérieure d'Electricité, Universités Paris VI et XI, URA \\ D 0127, Plateau du Moulon, 91192 Gif sur Yvette Cedex, France
}

(Reçu le 10 novembre 1989, révisé le 8 février 1990, accepté le 30 mars 1990)

\begin{abstract}
Résumé. - Une technique performante permettant de modéliser les champs magnétiques consiste à associer un calcul par éléments finis au sein des conducteurs et une méthode intégrale sur leur surface. Nous nous sommes intéressés ici à la partie éléments finis du calcul et nous comparons les précisions sur les champs et les courants induits ainsi que les temps de calcul lorsqu'on utilise trois types différents de variables : les champs aux nœuds, les projections sur chaque arête soit du champ au milieu de l'arête, soit des 2 champs en ses sommets.
\end{abstract}

\begin{abstract}
A performing technique that permits the modelling of magnetic fields consists in associating a finite element calculus inside the conductors and an integral method on their surface. We are interested here in the finite element calculus and we compare the precisions on the fields and induced currents as well as the computation times when three different types of variables are used : nodal fields, projections on the edges of the fields at the middle of the edges or at the vertices of the edges.
\end{abstract}

\section{Introduction.}

L'utilisation des éléments finis pour la modélisation en électromagnétisme donne lieu au développement de nombreuses méthodes. Cependant si l'on s'intéresse aux inconnues qui interviennent dans chacune d'elles, on peut classer ces méthodes en deux grands groupes : celles qui passent par l'intermédiaire des potentiels et celles qui calculent directement les grandeurs physiques, champs ou courants.

Parmi les premières, la plupart utilise le potentielvecteur A, associé éventuellement au potentiel scalaire électrique $\varphi[1,2]$. D'autres font appel au couple ( $\mathbf{T}, \boldsymbol{\Omega})[3,4]$, ou $\mathbf{T}$ est e c amp magnetique à grad $\Omega$ près. Un choix convenable de jauge permet de simplifier les calculs mais les champs sont obtenus par dérivation; ils sont donc connus avec une précision inférieure à celle des potentiels. Les codes bidimensionnels ont largement utilisé le potentiel-vecteur A dont l'intérêt est évident dans ce cas car il ne possède alors qu'une seule composante tandis que le champ qui en dérive en possède deux.

La formulation directe en termes de champ, associée à la mise en place d'un opérateur intégral (la rigidité extérieure), qui permet de réduire le domaine à mailler, représente une approche très puissante du problème. Elle a été exploitée dans le code Trifou [5] qui utilise comme degrés de liberté les circulations du champ magnétique le long des arêtes du maillage. Une formulation duale [6] en termes de champ électrique a été implémentée récemment [7]. Les fonctions de bases utilisées pour discrétiser ces deux formulations directes sont associées aux arêtes des tétraèdres (une fonction de base par arête) [8]. Elles conduisent à des champs dont la composante tangentielle est continue d'un tétraèdre à l'autre, mais pas la composante normale. Ainsi, la 1scretisation a optee ans ri ou, par icu 1eremen intéressante lorsqu'on veut calculer des courants de Foucault est moins bien adaptée à la détermination des champs. On peut cependant améliorer la précision sur les champs en associant deux fonctions de base, et donc deux degrés de liberté, à chaque arête [9]. Malheureusement cette amélioration est obtenue au prix d'un doublement du nombre d'inconnues et de la multiplication par un facteur 4 du nombre d'éléments non nuls de la matrice du système à résoudre. Le calcul s'en trouve considérablement alourdi. 
Une autre manière de discrétiser la formulation directe en champ est envisageable. Elle consiste à choisir comme degrés de liberté les composantes des champs aux nœuds du maillage. La continuité du champ au passage des facettes entre tétraèdres est alors assurée tout naturellement à l'intérieur d'un matériau (si la facette commune est située sur la surface de transition entre deux matériaux où le champ est discontinu physiquement, un choix convenable des degrés de liberté - $\mathbf{b}_{\mathrm{n}}$ et $\mathbf{h}_{\mathrm{t}}$ par exemple permet la modélisation [10]). Cette méthode conduit, comme celle qui associe deux degrés de liberté à chaque arête, à une bonne détermination des champs, mais le calcul est beaucoup plus léger. Il est, comme nous le verrons, comparable à celui mis en œuvre dans la méthode utilisant des fonctions d'arête à un degré de liberté.

Nous nous proposons ici de comparer, pour la formulation directe en champ magnétique, les précisions obtenues sur les champs et les courants avec les trois types de fonctions de base : celles d'arête à un degré de liberté, celles d'arête à deux degrés de liberté et les fonctions nodales.

Les bases théoriques de cet article ont été mises en place au cours de l'année 1987, lors d'un séjour des auteurs à la Direction des Etudes et Recherches d'Electricité de France [11].

\section{Rappel de la formulation en champ magnétique.}

Soit $\Omega$ un domaine conducteur simplement connexe (pour simplifier la présentation) et $\partial \Omega$ la frontière de $\Omega$. Soit par ailleurs, un inducteur $\Omega^{\text {s }}$, distinct de $\boldsymbol{\Omega}$, parcouru par une densité de courant $\mathbf{J}^{\mathrm{s}}$. Si $\boldsymbol{\Omega}$ n'existait pas, $\Omega^{\text {s }}$ créerait un champ $h^{\text {s }}$. La présence de $\Omega$ provoque un champ de réaction $h^{R}$. Il règne finalement un champ total $\mathbf{h}=\mathbf{h}^{\mathrm{s}}+\mathbf{h}^{\mathrm{R}}$ qui obéit aux équations de Maxwell, écrites ici dans le cadre de l'approximation électrotechnique :

$$
\begin{aligned}
\operatorname{rot} \mathbf{h} & =\mathbf{J} \\
\operatorname{rot} \mathbf{e} & =-\frac{\partial \mathbf{b}}{\partial t} \\
\operatorname{div} \mathbf{b} & =0 .
\end{aligned}
$$

La formulation variationnelle associée [5] met en jeu des champs d'essai $\mathbf{h}^{\prime}$ de carré sommable, dont le rotationnel est nul hors de $\Omega$ et de carré sommable dans $\Omega$. Si D est le domaine de calcul, de frontière $\partial \mathrm{D}$, on $\mathrm{a}$ :

$$
\begin{aligned}
\frac{\mathrm{d}}{\mathrm{d} t} \int_{\mathrm{D}} \mu \mathbf{h} \cdot \mathbf{h}^{\prime}+\int_{\partial \mathrm{D}}(\mathbf{n} \wedge \mathbf{e}) \cdot \mathbf{h}^{\prime}+ \\
\quad+\int_{\Omega} \rho \operatorname{rot} \mathbf{h} \cdot \operatorname{rot} \mathbf{h}^{\prime}=0 .
\end{aligned}
$$

La perméabilité $\mu$ du conducteur $\Omega$ est fonction de $|\mathbf{h}| ; \boldsymbol{\rho}$ est la résistivité de $\Omega, \mathbf{n}$ est la normale sortante de $\mathrm{D}$. La détermination de $\mathbf{h}$ nécessite la connaissance de e sur $\partial \mathrm{D}$, c'est pourquoi on n'utilise pas en général l'équation (1) mais celle qui en dérive en prenant pour D l'espace entier. A ce moment-là, l'intégrale de surface s'annule; de plus, hors de $\boldsymbol{\Omega}$, le champ de réaction $\mathbf{h}^{\mathrm{R}}$ et le champ d'essai $\mathbf{h}^{\prime}$ ont un rotationnel nul et dérivent donc de potentiels :

$$
\begin{aligned}
\mathbf{h}^{\mathrm{R}} & =\nabla \varphi \\
\mathbf{h}^{\prime} & =\nabla \varphi^{\prime} .
\end{aligned}
$$

L'équation variationnelle de base devient ainsi :

$$
\begin{array}{r}
\frac{\mathrm{d}}{\mathrm{d} t}\left[\int_{\Omega} \mu \mathbf{h} \cdot \mathbf{h}^{\prime}+\int_{\partial \Omega} \mu_{0}\left(\frac{\partial \varphi}{\partial n}+\mathbf{h}^{\mathrm{s}} \cdot \mathbf{n}\right) \varphi^{\prime}\right]+ \\
+\int_{\Omega} \rho \operatorname{rot} \mathbf{h} \cdot \operatorname{rot} \mathbf{h}^{\prime}=0 \quad \forall \mathbf{h}^{\prime} \in \mathrm{H}
\end{array}
$$

où $\quad H=\left\{h \in \mathbb{L}^{2}\left(\mathbb{R}^{3}\right): \quad\right.$ rot $h \in \mathbb{L}^{2}\left(\mathbb{R}^{3}\right), \quad$ rot $h=0$ dans $\left.\mathbb{R}^{3}-\Omega\right\}$

n est la normale sortante de $\Omega$.

Le traitement numérique de l'équation (2) nécessite en particulier la discrétisation de l'intégrale de surface $\int \frac{\partial \varphi}{\partial n} \varphi^{\prime}$ et le calcul de la matrice de rigidité extérieure reliant $\varphi$ et $\frac{\partial \varphi}{\partial n}$ [12].

\section{Représentation des champs.}

Pour discrétiser l'équation (1) (ou l'Eq. (2)), on maille le domaine $\mathrm{D}$ (ou le conducteur $\Omega$ ) en tétraèdres et par suite sa surface $\partial \mathrm{D}($ ou $\partial \Omega$ ) en triangles et on adopte une représentation linéaire du champ dans chaque tétraèdre pour les trois types de description du champ que nous voulons comparer. On appelle, dans tous les cas, $\lambda_{i}$ la coordonnée barycentrique associée au sommet $i$ [13].

Dans la description nodale $(\mathrm{N})$, on pose :

$$
\mathbf{h}_{\mathrm{N}}=\sum_{i=1}^{4} \mathbf{h}_{i} \lambda_{i}
$$

$\mathbf{h}_{i}$ étant le champ au nœud $i$. Le champ est ainsi continu d'un tétraèdre à l'autre.

Dans la description avec fonctions d'arête, on appelle $\{i j\}$ l'arête de sommets $i$ et $j$. Avec les éléments d'arête (A1), l'expression du champ dans un tétraèdre s'écrit :

$$
\mathbf{h}_{\mathrm{A} 1}=\sum_{\{i, j\}=1}^{6} \alpha_{i j}\left(\lambda_{i} \nabla \lambda_{j}-\lambda_{j} \nabla \lambda_{i}\right)
$$

$\lambda_{i} \nabla \lambda_{j}-\lambda_{j} \nabla \lambda_{i}$ est la fonction de base associée à l'arête $\{i j\}$. Il est facile de voir que :

$$
\alpha_{i j}=\frac{\mathbf{h}_{\mathrm{Al}, i}+\mathbf{h}_{\mathrm{Al}, j}}{2} \cdot \mathbf{i j} .
$$


Ainsi, le degré de liberté $\alpha_{i j}$ est lié à la projection du champ au milieu de l'arête $\{i, j\},\left(\mathbf{h}_{\mathrm{A} 1, i}+\mathbf{h}_{\mathrm{A} 1, j}\right) / 2$, sur cette arête.

Avec les fonctions à 2 degrés de liberté par arête, que nous appellerons éléments d'arête $\mathrm{A} 2$, on pose :

$$
\mathbf{h}_{\mathrm{A} 2}=\sum_{\{i, j\}=1}^{6} \beta_{i j} \lambda_{i} \nabla \lambda_{j}+\beta_{j i} \lambda_{j} \nabla \lambda_{i}
$$

on peut vérifier que :

$$
\boldsymbol{\beta}_{i j}=\mathbf{h}_{\mathrm{A} 2, i} \cdot \mathbf{i j}, \quad \boldsymbol{\beta}_{j i}=\mathbf{h}_{\mathrm{A} 2, j} \cdot \mathbf{j i} .
$$

Les coefficients $\beta_{i j}$ sont donc liés aux projections des champs aux sommets sur les arêtes qui aboutissent en ces sommets.

On peut aussi vérifier qu'avec les deux types d'éléments d'arête, $\mathrm{A} 1$ et $\mathrm{A} 2$, le champ tangentiel à la traversée d'une facette commune à deux tétraèdres est continu mais le champ normal ne l'est pas; le champ en un nœud dépend du tétraèdre considéré.

Dans les trois descriptions, le courant $\mathbf{J}=\operatorname{rot} \mathbf{h}$ est constant par tétraèdre. En remplaçant $h$ par (3), (4) ou (5), il vaut :

- pour $\mathrm{N}$ :

$$
\mathbf{J}=\sum_{i=1}^{4} \nabla \lambda_{i} \wedge \mathbf{h}_{i}
$$

- pour A1 :

$$
\mathbf{J}=\sum_{\{i, j\}=1}^{6} 2 \alpha_{i j} \nabla \lambda_{i} \wedge \nabla \lambda_{j}
$$

- pour A2:

$$
\mathbf{J}=\sum_{\{i, j\}=1}^{6}\left(\beta_{i j}-\beta_{j i}\right) \nabla \lambda_{i} \wedge \nabla \lambda_{j} .
$$

\section{Comparaison des représentations d'un champ connu.}

Soit un champ $\mathbf{h}_{\mathrm{c}}$ supposé connu sur un tétraèdre. $\mathrm{Si}$ le champ varie peu sur le tétraèdre, ce qui est le cas dans un maillage bien adapté, il sera bien approximé par son développement au $1^{\text {er }}$ ordre.

Dans ces conditions, le champ nodal construit par interpolation linéaire entre les valeurs connues aux sommets :

$$
\mathbf{h}_{\mathrm{N}}=\sum_{i=1}^{4} \mathbf{h}_{i} \lambda_{i}
$$

est égal au champ $\mathbf{h}_{\mathrm{c}}$ au second ordre près.

En calculant $\beta_{i j}$ à l'aide de (7) et en utilisant $\mathbf{h}_{\mathrm{c}}$ connu à la place de $\mathbf{h}_{\mathrm{A} 2}$ inconnu, on obtient :

$$
\begin{aligned}
\mathbf{h}_{\mathrm{A} 2}=\sum_{\{i, j\}=1}^{6}\left\{\left(\mathbf{h}_{\mathrm{c} i} \cdot \mathbf{i j}\right) \lambda_{i} \nabla \lambda_{j}+\right. & \\
& \left.+\left(\mathbf{h}_{\mathrm{cj}} \cdot \mathbf{j i}\right) \lambda_{j} \nabla \lambda_{i}\right\} .
\end{aligned}
$$

On en déduit [11], en projetant sur les 3 arêtes issues de $i$ par exemple :

$$
\mathbf{h}_{\mathrm{A} 2, i}=\mathbf{h}_{\mathrm{c} i} .
$$

Ainsi, le champ $\mathbf{h}_{\mathrm{A} 2}$ résulte d'une interpolation linéaire sur le tétraèdre entre les valeurs $\mathbf{h}_{\mathrm{c} i}$ aux 4 sommets et est par suite égal au champ $\mathbf{h}_{\mathrm{N}}$.

Pour la représentation $\mathrm{A} 1$, en calculant $\alpha_{i j}$ à partir de $\mathbf{h}_{\mathrm{c}}$, on a :

$\mathbf{h}_{\mathrm{A} 1}=\sum_{\{i, j\}=1}^{6}\left(\frac{\mathbf{h}_{\mathrm{c} i}+\mathbf{h}_{\mathrm{cj} j}}{2} \cdot \mathbf{i j}\right)\left(\lambda_{i} \nabla \lambda_{j}-\lambda_{j} \nabla \lambda_{i}\right)$

soit encore, en comparant avec (10):

$\mathbf{h}_{\mathrm{A} 1}=\mathbf{h}_{\mathrm{A} 2}-\sum_{\{i, j=1}^{6}\left(\frac{\mathbf{h}_{\mathrm{c} i}+\mathbf{h}_{\mathrm{c} i}}{2} \cdot \mathbf{i j}\right) \nabla\left(\lambda_{i} \lambda_{j}\right)$.

En particulier, au niveau du nœud $i$, compte tenu de (13) :

$$
\mathbf{h}_{\mathrm{A} 1, i}=\mathbf{h}_{\mathrm{c} i}-\sum_{i=1, i \neq i}^{4} \frac{\mathbf{h}_{\mathrm{c} i}-\mathbf{h}_{\mathrm{c} j}}{2} \cdot \mathbf{i j} \nabla \lambda_{j} .
$$

Le champ Al dépend du tétraèdre considéré. Pour un nœud donné, il n'est pas le même dans les différents tétraèdres ayant ce nœud pour sommet. Il diffère du champ connu et varie d'un tétraèdre à l'autre, d'une quantité qui est de l'ordre de grandeur de la variation du champ sur le tétraèdre.

Dans les trois représentations, les circulations $\int_{i}^{j} \mathbf{h} \cdot \mathrm{dl}$ sont égales; elles valent $\frac{1}{2}\left(\mathbf{h}_{\mathrm{c} i}+\mathbf{h}_{\mathrm{cj}}\right) \cdot \mathbf{i j}$ et par suite les courants induits, constants par tétraèdre, ont les mêmes valeurs.

Compte tenu des propriétés que nous venons de dégager concernant les trois représentations $\mathrm{A} 1, \mathrm{~A} 2$ et $\mathbf{N}$ d'un champ connu sur un tétraèdre, si l'on s'intéresse maintenant aux champs calculés numériquement sur un domaine il paraît $a$ priori raisonnable de penser que :

- les représentations $\mathrm{N}$ et $\mathrm{A} 2$ conduiront à deux approximations linéaires sensiblement équivalentes du champ (au moins pour le cas étudié où celui-ci est continu);

- la représentation A1 fournira une approximation nettement moins bonne;

constant par tétraèdre, avec une précision du même ordre.

Il s'agit à présent de vérifier si, lors de la résolution des équations de Maxwell, nous aboutissons effectivement à ces conclusions.

\section{Test numérique.}

Nous avons considéré un système dans lequel nous savions calculer analytiquement les champs et courants; nous avons alors résolu numériquement 
l'équation (1) à l'intérieur d'un domaine $\mathrm{D}$ en nous donnant les conditions aux limites (c'est-à-dire le champ électrique calculé analytiquement) sur sa frontière $\partial \mathrm{D}$. Le test sur l'équation (1) plutôt que sur l'équation (2) présente l'avantage de ne pas avoir à calculer de matrice de rigidité extérieure et donc de ne pas ajouter les erreurs correspondantes.

Le système testé est représenté figure 1. Le courant inducteur de densité $\mathbf{J}$, de pulsation $\omega$ circule parallèlement à $\mathrm{O} z$ dans une plaque infinie perpendiculaire à $\mathrm{O} y$, d'épaisseur $a_{2}-a_{1}$ :

$$
\mathbf{J}=\mathbf{J}_{0} \cos (\omega t-p x) .
$$

Sa densité varie sinusoïdalement le long de $\mathrm{O} x$, le nombre d'ondes étant $p$. Une plaque conductrice très épaisse de conductivité $\sigma$, de perméabilité $\mu_{0}$ est placée parallèlement à la plaque inductrice. Il s'y développe des courants induits parallèles à $\mathrm{O} z$.

Les diverses grandeurs physiques de ce système sont indépendantes de $z$. On peut facilement les déterminer par un calcul analytique. Passant en grandeurs complexes et posant :

$$
h=2 \operatorname{Re}\left[h_{0} \mathrm{e}^{i(\omega t-p x)}\right]
$$

on obtient, pour la région conductrice :

$$
\begin{aligned}
& h_{x 0}=i k A \mathrm{e}^{k p y} \\
& h_{y 0}=A \mathrm{e}^{k p y} \\
& h_{z 0}=0 \\
& e_{z 0}=-\frac{\mu_{0} \omega}{p} h_{y 0} \\
& e_{x 0}=e_{y 0}=0 \\
& J_{z 0}=\sigma e_{z 0}
\end{aligned}
$$

avec :

$$
\begin{gathered}
k=1+i \sigma \mu_{0} \omega / p^{2} \\
A=\frac{i\left|\mathbf{J}_{0}\right|}{p\left(\mu_{0}+k\right)}\left[\mathrm{e}^{-p a_{1}}-\mathrm{e}^{-p a_{2}}\right] .
\end{gathered}
$$

Nous avons effectué le calcul numérique du champ magnétique et du courant dans le parallélépipède $\mathrm{D}$, ayant pour longueur $\pi / 2 p$ le long de $\mathrm{O} x$ ( $1 / 4$ de longueur d'onde) ; $D$ est maillé en tétraèdres. Le champ électrique sur $\partial \mathrm{D}$ a été déterminé par (15).

Quatre exemples ont été considérés, correspondant à des variations très différentes des champs sur le maillage : dans tous les cas, on a pris $\sigma=5 \times$ $10^{7} \Omega . \mathrm{m}, \quad \omega=100 \mathrm{rad} / \mathrm{s}$ ce qui correspond à une épaisseur de peau $\delta=18 \mathrm{~mm}$. Les domaines D avaient pour hauteur et pour largeur $1 \mathrm{~mm}$ et leur longueur $\pi / 2 p$ était égale à $1,2,4$ ou $8 \mathrm{~mm}$ (exemples 1, 2, 3, 4). Ils étaient découpés en 1, 2, 4 ou 8 cubes, lesquels étaient ensuite subdivisés en 24 tétraèdres. Bien que l'épaisseur de peau soit grande, la variation rapide du courant inducteur de long de $\mathrm{O} x$ donne une variation importante du champ aussi bien suivant $\mathrm{O} x$ que suivant $\mathrm{O} y$ (par exemple lorsque sa valeur passe de 1 à 0 suivant $O x$, elle passe suivant $O y$ de 1 à 0,2 dans le cas 1 , et de 1 à 0,8 dans le cas 4). Dans le tableau $I$, nous indiquons pour les 4 exemples le nombre de tétraèdres du maillage ainsi que les quantités $\left|\Delta h_{\mathrm{a} x}\right|_{\mathrm{M}} /\left|h_{\mathrm{a} x}\right|_{\mathrm{M}}$ et $\left|\Delta h_{\mathrm{a} y}\right|_{\mathrm{M}} /\left|h_{\mathrm{a} y}\right|_{\mathrm{M}}$. Les quantités $\Delta h_{\mathrm{a} x, \mathrm{M}}$ et $\Delta h_{\mathrm{a} y, \mathrm{M}}$ représentent, pour les champs analytiques, les plus grandes variations de $h_{\mathrm{ax}}$ et $h_{\mathrm{a} y}$ observées sur les tétraèdres du maillage $\left(h_{\mathrm{a} z}\right.$ est nul et $\Delta h_{\mathrm{a} z}$ aussi). $\left|h_{\mathrm{a} x}\right|_{\mathrm{M}}$ et $\left|h_{\mathrm{a} y}\right|_{\mathrm{M}}$ sont les valeurs
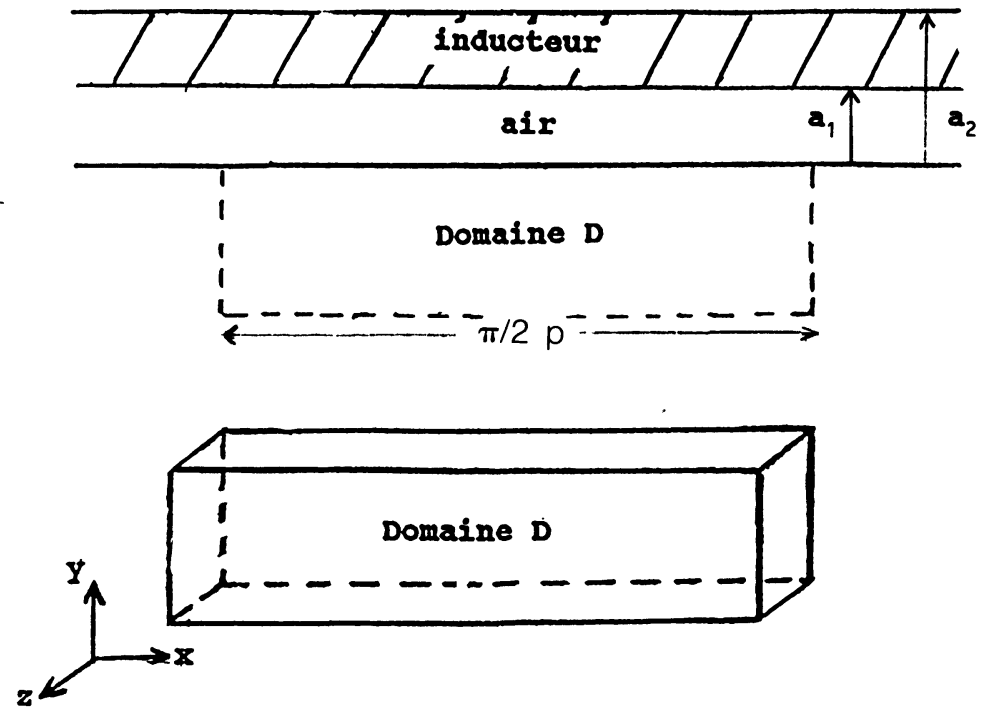

Fig. 1. - Schéma du système testé.

[Scheme of the tested system.] 
Tableau I. - Caractéristiques des exemples traités. [Characteristics of the considered examples.]

\begin{tabular}{|c|r|r|r|r|}
\hline Exemple & 1 & 2 & 3 & 4 \\
\hline Nombre tétraèdres & 24 & 48 & 96 & 192 \\
\hline $100\left|\Delta h_{\mathrm{a} x}\right|_{\mathrm{M}} /\left|h_{\mathrm{a} x}\right|_{\mathrm{M}}$ & 100 & 70 & 37 & 18 \\
\hline $100\left|\Delta h_{\mathrm{a} y}\right|_{\mathrm{M}} /\left|h_{\mathrm{a} y}\right|_{\mathrm{M}}$ & 80 & 54 & 32 & 17 \\
\hline
\end{tabular}

maximales de $h_{\mathrm{ax}}$ et $h_{\mathrm{ay}}$ sur D. Remarquons que $\left|\Delta J_{\mathrm{a} z}\right| /\left|J_{\mathrm{a} z}\right|_{M}$ est, d'après (15), égal à $\left|\Delta h_{\mathrm{a} y}\right|_{\mathrm{M}} /\left|h_{\mathrm{a} y}\right|_{\mathrm{M}}$. Le tableau I donne donc l'ordre de grandeur de la variation du champ ou du courant à l'intérieur d'un tétraèdre.

Pour les trois formulations et les quatre exemples, nous avons alors calculé :

- le champ au centre de gravité des tétraèdres, $h_{\mathrm{GN}}, h_{\mathrm{GA} 1}, h_{\mathrm{GA} 2}$;

- le champ aux nœuds du maillage (un par nœud en nodal, autant que de tétraèdres en chaque nœud pour les deux autres cas);

- le courant dans chaque tétraèdre;

et nous avons comparé ces grandeurs avec leurs valeurs analytiques. Plus précisément, pour les trois composantes $\boldsymbol{g}_{i x}, \boldsymbol{g}_{i y}, \boldsymbol{g}_{i z}$ de la grandeur locale $\mathbf{g}_{i}$ pour lesquelles nous déterminons les valeurs numériques $g_{i x \mathrm{n}}, g_{i y \mathrm{n}}, g_{i z \mathrm{n}}$ et nous connaissons les valeurs analytiques $g_{i x \mathrm{a}}, g_{i y \mathrm{a}}, g_{i z \mathrm{a}}$, nous avons calculé :

$$
\varepsilon_{\mathrm{r}}\left(g_{i x}\right)=\left|g_{i x \mathrm{n}}-g_{i x \mathrm{a}}\right| /\left|\mathbf{g}_{\mathrm{a}}\right|_{\mathrm{M}} \text { etc. }
$$

$\left|\mathbf{g}_{\mathrm{a}}\right|_{M}$ est la valeur maximale de $\left|\mathbf{g}_{\mathrm{a}}\right|$ sur $\mathrm{D}$ (nous avons défini $\varepsilon\left(g_{i x}\right)$ par référence à $\left|g_{\mathrm{a}}\right|_{M}$ plutôt qu'à partir de $g_{i \mathrm{a}}$ au point $i$ car celle-ci est nulle en certains points). Nous en avons déduit la valeur moyenne (en \%) de l'erreur :

$I$ est le nombre de valeurs déterminées pour $g_{i x}$ et sera précisé plus loin. Ces calculs ont été effectués séparément pour les parties réelle et imaginaire des composantes des champs et des courants.

Les erreurs trouvées étant pratiquement égales, par suite du choix du système étudié, nous n'avons indiqué, dans ce qui suit, que la valeur commune, par exemple pour $g_{x}: \varepsilon_{\mathrm{m}}\left(g_{x}\right)=\varepsilon_{\mathrm{m}}\left(\operatorname{Re}\left(g_{x}\right)\right)=$ $\varepsilon_{\mathrm{m}}\left(\operatorname{Im}\left(g_{x}\right)\right)$. De plus, nous avons calculé la valeur moyenne de l'erreur moyenne sur les trois composantes :

$$
\varepsilon_{\mathrm{m}}(\mathbf{g})=\left(\varepsilon_{\mathrm{m}}\left(g_{x}\right)+\varepsilon_{\mathrm{m}}\left(g_{y}\right)+\varepsilon_{\mathrm{m}}\left(g_{z}\right)\right) / 3 .
$$

Il faut bien remarquer que les définitions des erreurs sont les mêmes dans les trois formulations puisque les valeurs calculées sont comparées aux mêmes valeurs analytiques. Les résultats obtenus permetttent donc une comparaison objective de ces formulations.

Le tableau II donne les valeurs des erreurs moyennes sur les courants dans les trois formulations. La valeur calculée, constante sur chaque tétraèdre, a été comparée à la valeur analytique au centre de gravité. Nous constatons que la précision sur le courant est la même quelle que soit la formulation.

Tableau II. - Erreurs moyennes sur les courants (en $\%)$.

[Average errors on the currents (in \%).]

\begin{tabular}{|c|c|c|c|c|c|}
\hline Ex. & Form. & $\varepsilon_{\mathrm{m}}\left(\mathbf{j}_{\mathbf{r}}\right)$ & $\varepsilon_{\mathrm{m}}\left(\mathbf{j}_{y}\right)$ & $\varepsilon_{\mathrm{m}}\left(\mathbf{j}_{\mathbf{z}}\right)$ & $\varepsilon_{\mathrm{m}}(\mathbf{j})$ \\
\hline \multirow{3}{*}{1} & $\mathrm{~A} 1$ & 3,3 & 3,3 & 2,8 & 3,1 \\
& $\mathrm{~A} 2$ & 3,2 & 3,5 & 2,4 & 3,0 \\
& $\mathrm{~N}$ & 1,9 & 4,9 & 3,7 & 3,5 \\
\hline \multirow{3}{*}{2} & $\mathrm{~A} 1$ & 2,2 & 2,3 & 1,5 & 2,0 \\
& $\mathrm{~A} 2$ & 2,2 & 2,3 & 1,4 & 2,0 \\
& $\mathrm{~N}$ & 1,6 & 3,1 & 2,0 & 2,2 \\
\hline \multirow{3}{*}{3} & $\mathrm{~A} 1$ & 1,3 & 1,4 & 0,8 & 1,2 \\
& $\mathrm{~A} 2$ & 1,3 & 1,4 & 0,8 & 1,2 \\
& $\mathrm{~N}$ & 1,0 & 1,7 & 1,0 & 1,2 \\
\hline \multirow{3}{*}{4} & $\mathrm{~A} 1$ & 0,7 & 0,7 & 0,5 & 0,6 \\
& $\mathrm{~A} 2$ & 0,7 & 0,8 & 0,5 & 0,7 \\
& $\mathrm{~N}$ & 0,6 & 0,9 & 0,5 & 0,7 \\
\hline
\end{tabular}

(Le calcul numérique étant $3 \mathrm{D}$, nous avons calculé les trois composantes du courant et nous avons trouvé, pour les composantes en principe nulles $j_{x}$ et $j_{y}$, une faible valeur du même ordre de grandeur autres, un résultat relatif aux variables d'arête [14] : si dans une formulation $\mathrm{A} 1$, on remplace les variables A1 par des variables A2 dans les endroits où le champ varie rapidement, la précision sur le champ devient meilleure mais celle sur le courant n'est pas améliorée. Il est à remarquer que la précision sur le courant est excellente, car l'erreur $\varepsilon_{\mathrm{m}}(\mathbf{J})$ est bien inférieure à la variation du courant d'un tétraèdre à l'autre.

Nous avons par ailleurs considéré les champs nodaux. La figure 2 montre, pour un nœud particu- 


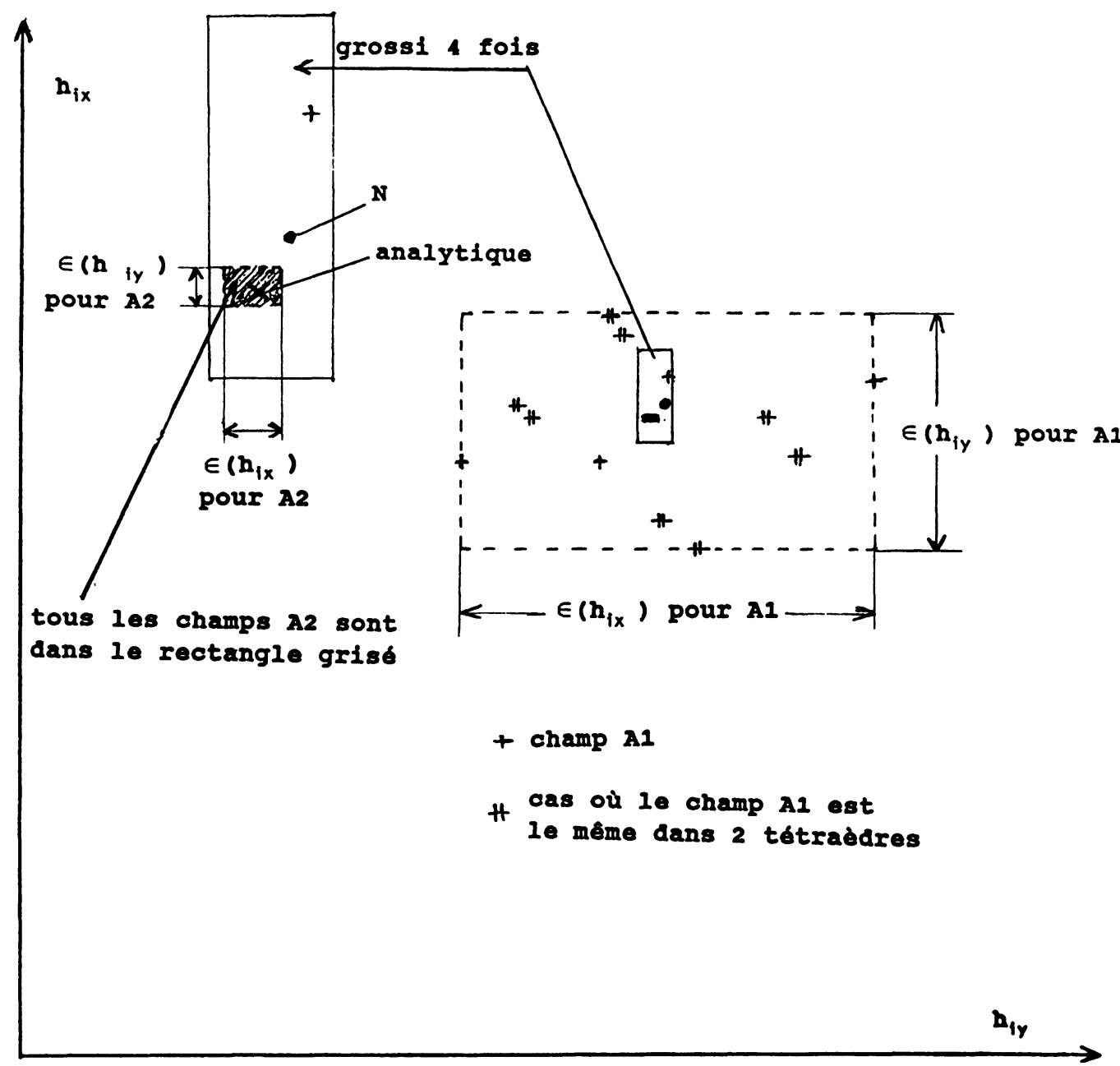

Fig. 2. - Les différentes valeurs numériques de la partie $\left(h_{i x}, h_{i y}\right)$ du champ au nœud $i$ dans les tétraèdres ayant $i$ pour sommet.

[The different numerical values of the $\left(h_{i x}, h_{i y}\right)$ part of the field at the node $i$ in the tetrahedrons for which $i$ is a vertex.]

lier de l'exemple 3, les différentes déterminations numériques des composantes $h_{x}$ et $h_{y}$ à $t=0$ dans les trois formulations $\mathrm{A} 1, \mathrm{~A} 2$ et $\mathrm{N}$. On voit que les champs $\mathrm{Al}$ sont très dispersés; ici, les diverses valeurs entourent la valeur analytique, mais ce n'est pas toujours le cas. Les champs A2 sont tous à l'intérieur du petit rectangle indiqué, dont les dimensions sont du même ordre que l'écart entre le champ $\mathbf{N}$ et le champ analytique.

Le tableau III est relatif aux champs nodaux. Comme le champ en un nœud a, dons les formulations $\mathrm{A} 1$ et $\mathrm{A} 2$, autant de déterminations qu'il existe de tétraèdres ayant ce nœud comme sommet, nous avons défini l'erreur en chaque nœud comme l'écart entre les valeurs extrêmes obtenues pour chaque composante de champ, soit pour $h_{i x}$ (cf. Fig. 2) :

$$
\varepsilon\left(h_{i x}\right)=\left[h_{i x}\right]_{\text {Max }}-\left[h_{i x}\right]_{\min } .
$$

Nous avons ensuite pris la moyenne (en \%) sur l'ensemble des $N$ nœuds :

$$
\varepsilon_{\mathrm{m}}\left(h_{x}\right)=100 \sum_{i=1}^{N} \varepsilon\left(h_{i x}\right) / N\left|h_{\mathrm{ax}}\right|_{\mathrm{Max}} .
$$

Tableau III. - Erreurs moyennes sur les champs aux nouds (en \%)

[Average errors on the fields at the nodes (in \%).]

\begin{tabular}{|c|c|r|r|r|r|}
\hline Ex. & Form. & $\varepsilon_{\mathrm{m}}\left(h_{\mathrm{n} .}\right)$ & $\varepsilon_{\mathrm{m}}\left(h_{\mathrm{n} !}\right)$ & $\varepsilon_{\mathrm{m}}\left(h_{\mathrm{n}=}\right)$ & $\varepsilon_{\mathrm{m}}\left(h_{\mathrm{n}}\right)$ \\
\hline \multirow{4}{*}{1} & $\mathrm{~A} 1$ & 29,7 & 27,4 & 20,2 & 25,8 \\
& $\mathrm{~A} 2$ & 13,1 & 11,6 & 10,6 & 11,8 \\
& $\mathrm{~N}$ & 10,3 & 10,4 & 4,8 & 8,5 \\
\hline \multirow{4}{*}{2} & $\mathrm{~A} 1$ & 31,7 & 26,3 & 18,1 & 25,4 \\
& $\mathrm{~A} 2$ & 5,6 & 5,4 & 3,8 & 4,9 \\
& $\mathrm{~N}$ & 3,2 & 5,2 & 4,0 & 4,1 \\
\hline \multirow{3}{*}{3} & $\mathrm{~A} 1$ & 22,5 & 18,2 & 11,3 & 17,3 \\
& $\mathrm{~A} 2$ & 1,6 & 1,5 & 1,1 & 1,4 \\
& $\mathrm{~N}$ & 1,0 & 2,0 & 1,9 & 1,6 \\
\hline \multirow{4}{*}{4} & $\mathrm{~A} 1$ & 13,6 & 10,9 & 6,5 & 10,3 \\
& $\mathrm{~A} 2$ & 0,5 & 0,4 & 0,3 & 0,4 \\
& $\mathrm{~N}$ & 0,6 & 0,9 & 0,8 & 0,8 \\
\hline \multirow{6}{*}{} & & & & & \\
\hline
\end{tabular}


Là encore, les erreurs étant quasiment identiques pour les parties réelle et imaginaire, nous n'avons indiqué dans le tableau que la valeur commune; elle caractérise l'ordre de grandeur des sauts des champs à la traversée des facettes.

De plus, nous avons calculé en chaque nœud la moyenne $\overline{h_{i \mathrm{n}}}$ des champs A1 et celle des champs A2 (sur la Fig. 2, ce sont les barycentres des nuages de points) puis nous avons comparé ces moyennes avec les champs analytiques. Le tableau IV donne les résultats.

Tableau IV. - Erreurs moyennes sur les champs aux nauds "moyennés" (en \%).

[Average errors on the "averaged" fields at the nodes (in \%).]

\begin{tabular}{|c|c|c|c|c|c|}
\hline Ex. & Form. & $\varepsilon_{\mathrm{m}}\left(h_{\mathrm{n} .}\right)$ & $\varepsilon_{\mathrm{m}}\left(h_{\mathrm{n} 1}\right)$ & $\varepsilon_{\mathrm{m}}\left(h_{\mathrm{n} z}\right)$ & $\varepsilon_{\mathrm{m}}\left(h_{\mathrm{r}}\right)$ \\
\hline \multirow{2}{*}{1} & A1 & 22,5 & 22,5 & 8,4 & 17,8 \\
& $\mathrm{~A} 2$ & 4,3 & 4,2 & 2,8 & 3,8 \\
\hline \multirow{2}{*}{2} & $\mathrm{~A} 1$ & 15,0 & 15,3 & 6,2 & 12,0 \\
& $\mathrm{~A} 2$ & 1,6 & 1,6 & 1,1 & 1,4 \\
\hline \multirow{2}{*}{3} & $\mathrm{~A} 1$ & 8,8 & 8,9 & 3,8 & 7,2 \\
& $\mathrm{~A} 2$ & 0,5 & 0,4 & 0,3 & 0,4 \\
\hline \multirow{2}{*}{4} & $\mathrm{~A} 1$ & 5,0 & 5,0 & 2,2 & 4,0 \\
& $\mathrm{~A} 2$ & 0,6 & 0,15 & 0,1 & 0,3 \\
\hline
\end{tabular}

Enfin le tableau $\mathrm{V}$ donne les erreurs moyennes sur les champs aux centres de gravité des tétraèdres.

L'ensemble de ces tableaux nous montre que :

- Dans la formulation $\mathbf{N}$ et encore plus dans les formulations $\mathrm{A} 1$ et $\mathrm{A} 2$, les champs aux centres de gravité sont connus de façon nettement plus précise que ceux aux nœuds. La pondération de Galerkine favorise les barycentres; les représentations graphiques utilisant ces valeurs sont donc les meilleures possibles pour le champ.

- Les sauts des champs nodaux A2 et les imprécisions sur les champs nodaux $\mathbf{N}$ sont du même ordre, très inférieurs à la variation du champ dans un tétraèdre. Les sauts des champs nodaux A1 sont beaucoup plus grands que ceux pour A2 ; ils sont comparables aux variations du champ dans un tétraèdre.

- Les valeurs moyennes des champs nodaux A1 et A2 sont mieux connues que les champs euxmêmes, mais ces champs nodaux moyennés ne
Tableau V. - Erreurs moyennes sur les champs aux centres de gravité (en \%).

[Average errors on the fields at the center of gravity (in \%).]

\begin{tabular}{|c|c|c|c|c|c|}
\hline Ex. & Form. & $\varepsilon_{\mathrm{m}}\left(h_{\mathrm{g}}\right)$ & $\varepsilon_{\mathrm{m}}\left(h_{\mathrm{g} 1}\right)$ & $\varepsilon_{\mathrm{m}}\left(h_{\mathrm{g}=}\right)$ & $\varepsilon_{\mathrm{m}}\left(h_{\mathrm{g}}\right)$ \\
\hline \multirow{3}{*}{1} & $\mathrm{~A} 1$ & 8,8 & 9,0 & 6,7 & 8,2 \\
& $\mathrm{~A} 2$ & 0,7 & 0,8 & 0,6 & 0,7 \\
& $\mathrm{~N}$ & 4,5 & 4,7 & 1,9 & 3,7 \\
\hline \multirow{3}{*}{2} & $\mathrm{~A} 1$ & 5,8 & 6,3 & 5,0 & 5,7 \\
& $\mathrm{~A} 2$ & 0,3 & 0,3 & 0,2 & 0,3 \\
& $\mathrm{~N}$ & 1,3 & 2,2 & 1,7 & 1,7 \\
\hline \multirow{3}{*}{3} & $\mathrm{~A} 1$ & 3,5 & 3,7 & 3,0 & 3,4 \\
& $\mathrm{~A} 2$ & 0,18 & 0,09 & 0,06 & 0,1 \\
& $\mathrm{~N}$ & 0,4 & 1,1 & 1,0 & 0,8 \\
\hline \multirow{4}{*}{4} & $\mathrm{~A} 1$ & 2,1 & 2,1 & 1,7 & 2,0 \\
& $\mathrm{~A} 2$ & 0,35 & 0,06 & 0,02 & 0,16 \\
& $\mathrm{~N}$ & 0,4 & 0,5 & 0,5 & 0,5 \\
\hline
\end{tabular}

peuvent être utiles qu'au point de vue graphique et non au point de vue numérique. En effet toute grandeur, par exemple une force, s'obtiendra à partir d'un calcul qui sera effectué élément de maillage par élément de maillage, et donc à partir des champs non moyennés.

\section{Importance des calculs.}

La majeure partie du temps de calcul est consacré à la résolution du système linéaire obtenu après discrétisation de l'équation (2). Il est donc essentiel de préciser les caractéristiques de la matrice correspondant à ce système.

Soient $N_{\mathrm{S}}$ le nombre de sommets dans $\mathrm{D}$ et $N_{\mathrm{A}}$ le nombre d'arêtes. Le nombre de variables mis en jeu est :

$$
\begin{aligned}
& -3 N_{\mathrm{s}} \text { dans } \mathrm{N}, \\
& -N_{\mathrm{A}} \text { dans } \mathrm{A} 1, \\
& -2 N_{\mathrm{A}} \text { dans } \mathrm{A} 2 \text {. }
\end{aligned}
$$

de calcul sont du même ordre; ils sont beaucoup plus élevés pour A2. Il est facile d'étendre ces conclusions à un maillage comportant beaucoup plus d'éléments. En effet, pour un système où la majeure partie des sommets du maillage sont des sommets internes, on a: $N_{\mathrm{A}} \sim 6 N_{\mathrm{S}}$. Le nombre de variables mis en jeu est donc 2 fois plus important dans A1 que $\mathbf{N}$ et quatre fois plus dans $\mathrm{A} 2$ que $\mathrm{N}$. La matrice du système est creuse. Elle comporte :

- environ 19 éléments par ligne pour A1 (puisqu'une arête est liée par l'intermédiaire d'un 
tétraèdre à elle-même et à 18 autres arêtes environ) et 2 fois plus pour A2 (puisqu'il y a 2 fonctions par arête au lieu d'une dans A1);

- environ 39 pour le nodal, puisqu'un sommet est lié à lui-même et à environ 12 autres et qu'il y a 3 inconnues par nœud.

Le nombre d'éléments non nuls total de la matrice est donc: $19 N_{\mathrm{A}} \sim 114 N_{\mathrm{S}}$ pour $\mathrm{A} 1,38 \times 2 N_{\mathrm{A}} \sim$ $456 N_{\mathrm{S}}$ pour $\mathrm{A} 2$ et $39 \times 3 N_{\mathrm{S}}=117 N_{\mathrm{S}}$ pour N. Il est du même ordre pour $\mathrm{N}$ et $\mathrm{A} 1,4$ fois plus important pour A2. Or, dans les méthodes itératives habituellement utilisées pour résoudre ce type de système, l'importance du calcul pour chaque itération dépend essentiellement du nombre d'éléments non nuls de la matrice : il est donc du même ordre pour $\mathrm{N}$ et $\mathrm{A} 1$, très nettement plus lourd pour $\mathrm{A} 2$. Il reste à évaluer l'influence de chaque formulation sur le nombre d'itérations dans le cas où $N_{\mathrm{S}}$ et $N_{\text {A }}$ sont grands.

\section{Conclusion.}

La modélisation des champs magnétiques à partir de la formulation directe en champ est particulièrement intéressante en 3D. Elle associe un calcul par éléments finis au sein des conducteurs à une méthode par intégrale de frontière à la surface de ceux-ci. Nous nous sommes intéressés ici au premier calcul et avons comparé les résultats obtenus lorsqu'on utilise trois types différents de variables: les champs aux nœuds $(\mathrm{N})$, les projections sur les arêtes des champs aux milieux des arêtes (A1) ou aux sommets des arêtes (A2).

Nous avons constaté sur les exemples traités que, dans les trois cas, le courant induit, constant par tétraèdre, est obtenu avec la même précision. Les champs, linéaires par tétraèdre, sont aussi déterminés avec la même précision pour $\mathrm{N}$ et $\mathrm{A} 2$, mais au prix d'un calcul beaucoup plus lourd pour A2 (4 fois plus d'éléments dans la matrice du système). Les champs A1 sont les moins bien déterminés, le calcul étant de la même importance qu'avec $\mathbf{N}$.

En conclusion, lorsqu'on ne tient compte que de la partie du calcul effectuée à l'intérieur du conducteur, la formulation $\mathbf{N}$ apparaît plus intéressante puisqu'elle associe la précision de $\mathrm{A} 2$ et la rapidité de calcul de A1. Les tests effectués ici l'ont été avec un matériau non ferromagnétique $\left(\mu=\mu_{0}\right)$. Dans une étape ultérieure, nous comparerons les méthodes dans le cas d'une perméabilité $\mu(|h|)$, en particulier en utilisant pour $\mathrm{N}$ une description linéaire de $b$ et $h$ dans chaque tétraèdre [10]. Mais auparavant, nous effectuerons la comparaison pour $\mu=\mu_{0}$ en associant des éléments finis dans le conducteur et une intégrale de frontière sur leur surface ; les méthodes avec variables d'arête présentent alors l'avantage de ne pas avoir à définir de normale aux surfaces et il pourra éventuellement être intéressant d'associer des variables nodales dans les conducteurs et des variables d'arêtes sur leur surface. Les comparaisons globales permettront de préciser les domaines d'utilisation de chaque méthode.

\section{Bibliographie}

[1] EMSON C. R. I., Simkin J., An optimal method for 3D eddy-currents, IEEE Trans. MAG-19 (1983) 2450-2452.

[2] Coulomb J. L., Finite element three-dimensional magnetic field computation, IEEE Trans. MAG17 (1981) 3241-3246.

[3] Carpenter C. J., Comparison of alternative formulations of 3-dimensional magnetic-field and eddy-current problems at power frequencies, Proc. IEE 124 (1977) 1026-1034.

[4] Bouillault F., Ren Z., Razek A., 3D eddycurrent $\mathrm{T}-\boldsymbol{\Omega}$ formulation integrals method, IEEE Trans. $M A G$ à paraître (1990)

[5] Bossavit A., Vérité J. C., The Trifou code: solving the $3 \mathrm{D}$ eddy-current problems by using $\mathrm{h}$ as state variable, IEEE Trans. MAG-17 (1983) 2465-2470.

[6] Bossavit A., Le calcul des courants de Foucault en dimension 3, avec le champ électrique comme inconnue, Revue Phys. Appl. (même numéro) 25 (1990).

[7] Ren Z., Bouillault F., Razek A., Bossavit A. and VÉRITÉ J. C., A new hybrid model using electric field formulation for 3D eddy current problems, à paraître, IEEE Mag 25 (mars 1990).
[8] NÉdelec J. C., Mixed finite elements in $\mathbb{R}^{3}$, Numer. Math. 35 (1980) 315-341.

[9] MUR G., DE Hopp A. T., A finite-element method for computing three-dimensional electromagnetic fields in inhomogeneous media, IEEE Trans. MAG-24 (1988) 330-333.

[10] Rioux-Damidau F., Nodal elements for threedimensional magnetic fields computation in inhomogeneous media, IEEE Trans. $M A G$ à paraître (1990).

[11] Rioux-Damidau F., Bandelier B., Modélisation tridimensionnelle des champs électromagnétiques dans le cadre d'une approximation linéaire nodale, EDF, Note interne HI/70-5962 (avril 1988).

[12] VÉrité J. C., Trifou : un code de calcul tridimensionnel des courants de Foucault, EDF, Bulletin de la DER, Série C, n 2 (1983) 79-92.

[13] Dhatt G., Touzot G., Une présentation de la méthode des éléments finis (Maloine éd.) 1984.

[14] Ren Z., VÉrité J. C., Application of a new edge element for 3D eddy-current computation, Proceedings Beijing International Symposium (Pergamon Press) octobre 1988, pp. 596-599. 